Nicolas de Aqueville, Sermones moralissimi atque ad populum instruendum utilissimi supra evangelia dominicarum totius anni

Marjorie Burghart

\title{
C OpenEdition
}

Journals

Édition électronique

URL : https://journals.openedition.org/ccm/6908

DOI : $10.4000 / \mathrm{ccm} .6908$

ISSN : 2119-1026

Éditeur

Centre d'études supérieures de civilisation médiévale/Université de Poitiers

Édition imprimée

Date de publication : 1 janvier 2021

Pagination : 86-87

ISBN : 978-2-490783-08-3

ISSN : 0007-9731

Référence électronique

Marjorie Burghart, « Nicolas de Aqueville, Sermones moralissimi atque ad populum instruendum utilissimi supra evangelia dominicarum totius anni », Cahiers de civilisation médiévale [En ligne], 253 | 2021, mis en ligne le 01 mars 2021, consulté le 23 novembre 2022. URL : http://journals.openedition.org/ccm/6908 ; DOI : https://doi.org/10.4000/ccm.6908

\section{(c) $(1)(9)$}

Creative Commons - Attribution - Pas d'Utilisation Commerciale - Pas de Modification 4.0 International - CC BY-NC-ND 4.0

https://creativecommons.org/licenses/by-nc-nd/4.0/ 
Nicolas de Aqueville, Sermones moralissimi atque ad populum instruendum utilissimi supra evangelia dominicarum totius anni, Eva Odelman (éd. et trad.), Turnhout, Brepols (Corpus Christianorum. Continuatio Mediaevalis, 283), 2018.

De Nicolaus de Aquaevilla, ou de Hacqueville, nous savons peu de choses : probablement originaire de Normandie, il se serait illustré vers la fin du XIII ${ }^{\mathrm{e}} \mathrm{s}$. par sa prédication à Lyon tout en entretenant des liens avec Paris. De cette activité nous conservons pour trace une collection de sermons modèles De tempore, les Sermones moralissimi, qui couvrent très classiquement un cycle allant du premier dimanche de l'Avent au XXVI ${ }^{\mathrm{e}}$ dimanche après la Pentecôte. Cette collection a joui d'un succès important et durable puisqu'elle nous est parvenue grâce à une cinquantaine de manuscrits, huit éditions incunables et pas moins de onze éditions plus récentes s'étalant jusqu'au XVII ${ }^{e}$ s. (1630). La collection semble notamment avoir été populaire en Suède médiévale, où les frères de Vadstena y ont ajouté des citations des Révélations de Brigitte - une version «augmentée» qui nous est parvenue grâce à trois manuscrits aujourd'hui conservés à la bibliothèque universitaire d'Uppsala (manuscrits C319, C320 et C322, étudiés par Monica Hedlund). Les soixante textes qui la composent et où, sous le latin, affleurent parfois quelques traces de français, respectent la structure typique de ce genre : chaque sermon débute par un thema biblique, qui est ensuite divisé puis subdivisé à l'envi. En tant que collection modèle, cet ensemble de sermons était destiné à être utilisé de manière pratique, notamment par des prédicateurs débutants ou en mal d'inspiration. Cet aspect pratique est reflété dans la collection par des renvois internes, indiquant $\mathrm{au}$ lecteur où trouver un passage sur un sujet connexe, ou d'intérêt complémentaire. Leur nature de modèle justifie aussi le caractère assez abstrait du contenu des sermons : destinés à fournir matière à prêcher dans toute la Chrétienté, ils contiennent très peu d'allusions aux lieux ou communautés effectivement fréquenté(e)s par leur auteur.

L'a. s'est attachée à offrir aux lecteurs modernes une édition fidèle de cette collection, accordant une part importante à la réflexion méthodologique qu'elle expose en introduction. Les sermons modèles étaient des textes «utilitaires», destinés à être au service d'un grand nombre de prédicateurs plutôt qu'à exprimer une pensée personnelle de l'auteur. Pour cela, ces textes pouvaient faire l'objet de multiples variations ou révisions lors de leur circulation, rendant extrêmement difficile le travail d'édition critique, et la méthode lachmannienne quasiment inopérante. De manière pragmatique, l'a. a donc choisi l'option d'une édition «semi-critique», dont le but n'est pas de retrouver le texte authentique tel qu'il aurait été composé par l'auteur, mais de rendre compte d'une version du texte tel qu'il a largement circulé et été utilisé, accompagnée d'un apparat restreint, n'enregistrant que les variantes réellement importantes. Pour cela, en conformité avec les recommandations d'un article méthodologique d'Antoine Dondaine ( Variantes de l'apparat critique dans les éditions de textes latins médiévaux ", Bulletin de la Société internationale pour l'étude de la philosophie médiévale, 4, 1962, p. 82-100, DOI : 10.1484/J.BPM.3.25), elle a basé son édition sur un unique «bon manuscrit» (ou plutôt un «bon témoin» puisqu'il s'agit en l'occurrence d'un incunable), en complémentant cette version par les informations fournies par une sélection d'autres témoins parmi ceux disponibles. Cette méthode d'édition «semi-critique» correspond plus ou moins à celle de la «transcription critique» telle que l'a décrite David D'Avray (Death and the Prince: Memorial Preaching before 1350, Oxford, Clarendon Press, 1994, p. 9-10), où l'effort investi doit être proportionnel au gain intellectuel qui en est espéré. Pour des raisons pratiques (facilité de lecture et d'accès) comme scientifiques (version du texte qui a joui théoriquement d'une plus grande circulation grâce à l'impression - à une date certes postérieure à l'activité principale de l'auteur), l'a. a choisi d'effectuer la transcription de base du texte d'après une édition 
incunable (Paris, pas avant 1477). Dans un travail préliminaire, l'a. a effectué deux éditions de test d'un sermon. L'une, stemmatique, était basée sur environ trente manuscrits parmi la cinquantaine connus. L'autre, semi-critique, était basée sur l'incunable mentionné plus haut, en utilisant dix manuscrits, dont deux seulement ont finalement été choisis pour figurer dans l'apparat, au moyen de tableaux de West. Une comparaison du résultat de ces deux éditions a montré que les différences étaient insignifiantes, justifiant le choix d'opter pour la méthode semi-critique, la moins coûteuse en temps.

L'édition telle qu'elle nous est présentée est basée sur deux exemplaires de la même édition incunable (Paris, pas avant 1477), le manuscrit identifié par l'a. comme ayant servi de source directe à cette édition incunable (Paris, BnF, Latin 15957) et deux manuscrits représentant des branches différentes de la tradition, comme établi lors des éditions de test (Beaune, Bibliothèque municipale, 48, et Londres, British Library, Add. 33416). Dans la liste des manuscrits et l'apparat, le choix des sigles est quelque peu original, en particulier pour les lecteurs habitués à réserver les lettres minuscules aux éditions anciennes : les sigles des manuscrits collationnés ont été attribués en fonction de l'ordre de réception des reproductions en microfilm, en parcourant d'abord l'alphabet en majuscules (y compris trois lettres suédoises), puis ce même alphabet en lettres minuscules

Parmi les soixante sermons présents dans l'édition incunable et son exemplar manuscrit, quatre sermons et un de leurs prothèmes ne se trouvent que dans ces témoins, à l'exclusion de la trentaine d'autres collationnés (sermons XIII, XIIII avec son prothema, LIII et LVII). Ces sermons, qui viennent explicitement s'ajouter aux précédents comme l'indiquent les mentions de eodem ou additio, sont d'une tonalité qui les démarque du reste de la collection : on y trouve des références géographiques et culturelles liées à Paris et au nord de la France, et le style en est plus simple, plus nourri d'exempla, avec par ailleurs un affleurement très présent du français à travers des mots à peine latinisés et même l'article $l y$. L'a., même si elle admet qu'il est très possible que ces textes ne soient pas de la plume de Nicolas de Hacqueville, a choisi de leur laisser leur place dans la collection, en particulier pour illustrer la manière dont les sermons modèles pouvaient être adaptés, amplifiés et appropriés par leurs utilisateurs.

La substance des sermons est conforme au contenu habituel des collections de modèles, entre exhortations au Salut et condamnation du péché et de ses périls. On y rencontre cependant quelques éléments témoignant d'une attention aux problèmes plus sociaux, en particulier à travers la condamnation de l'attitude de certains prélats, mais toujours très brièvement. Quant aux autorités, outre la Bible et sa Glose ordinaire, les sermons font la part belle aux sources patristiques (Augustin et Grégoire le Grand en particulier), et à Bernard de Clairvaux (lui-même, ou un Ps.-Bernard). L'usage de florilèges, non identifiés, semble confirmé par les fréquentes erreurs dans l'attribution des citations.

L'édition elle-même est complétée par un appendice (p. 587-596), qui donne la transcription d'une longue addition au sermon LI présente uniquement dans l'un des manuscrits utilisés (celui de Londres). Deux index terminent l'ouvrage : citations bibliques, et sources on peut au passage regretter l'absence d'un index des exempla, ou tout du moins d'une discussion de ceux-ci dans l'introduction, même si leur usage semble très modique dans la collection. Les interprétations des noms, auxquelles Nicolaus de Aquaevilla paraît avoir volontiers recours en appui à ses développements, pourraient également faire l'objet d'un index. S'il utilise les exempla avec parcimonie, Nicolaus semble avoir fait un usage intensif de la distinctio pour structurer ses sermons, en accord avec son temps. Dans l'apparat des sources, l'a. indique des renvois (cfr.) à des œuvres qui présentent des similitudes avec le passage concerné, sans pour autant en être nécessairement une source directe. Il ne faudra donc pas s'étonner de trouver dans ce dernier index des auteurs nettement postérieurs à la période d'activité de Nicolas de Hacqueville (comme par ex. Jean Hus). Si ce parti pris peut surprendre, il permet néanmoins de porter à la connaissance des lecteurs l'écho de certaines idées à travers les siècles, dans le temps long qui a été celui de la circulation de cette collection.

En conclusion, le travail proposé ici par l'a. donne à lire une importante collection de sermons. On peut certes regretter quelques points, comme l'absence d'informations sur Nicolaus de Aquaevilla dans l'introduction, sa biographie se réduisant à quelques très brefs éléments dans une note de bas de page, ou encore un passage un peu rapide sur l'explication des stratégies d'identification des sources. Mais en tout cas, le choix pragmatique d'une édition «semicritique» est en parfaite cohérence avec le genre du sermon modèle. Comme le recommandait déjà le P. Bataillon dès les années 1980, l'important est avant tout de donner à lire un texte tel qu'il a circulé et a été utilisé, plus que de reconstruire un hypothétique Urtext. On ne peut qu'espérer la multiplication de telles éditions, car au regard de l'ampleur du stock documentaire les sermons latins restent finalement très peu édités.

Marjorie BURGHART CNRS - UMR 5648 CIHAM 